\title{
A Junction Electric Field Determination of a Bifacial Silicon Solar Cell under a Constant Magnetic Field Effect by Using the Photoconductivity Method
}

\author{
Amadou Diao1, Bada Thiaw², Mountaga Boiro', Senghane Mbodji², Gregoire Sissoko \\ ${ }^{1}$ Semiconductors and Solar Energy Laboratory, Cheikh Anta Diop University, Dakar, Senegal \\ ${ }^{2}$ Research Team in Renewable Energies, Materials and Laser, Alioune Diop University, Bambey, Senegal \\ Email:ama_diao@yahoo.fr, amadou.diao@ucad.edu.sn
}

How to cite this paper: Diao, A., Thiaw, B., Boiro, M., Mbodji, S. and Sissoko, G. (2021) A Junction Electric Field Determination of a Bifacial Silicon Solar Cell under a Constant Magnetic Field Effect by Using the Photoconductivity Method. Journal of Modern Physics, 12, 635-645.

https://doi.org/10.4236/jmp.2021.125041

Received: March 17, 2021

Accepted: April 23, 2021

Published: April 26, 2021

Copyright $\odot 2021$ by author(s) and Scientific Research Publishing Inc. This work is licensed under the Creative Commons Attribution International License (CC BY 4.0).

http://creativecommons.org/licenses/by/4.0/

\begin{abstract}
In this work, a theory based on the steady photoconductivity method, of a bifacial silicon solar cell under polychromatic illumination and a magnetic field effect, is presented. The resolution of the continuity equation in the base of the solar cell, allowed us to establish the expression of the minority carriers' density from which the photoconductivity, the photocurrent density, the photovoltage and the solar output power as function of the junction recombination velocity and the applied magnetic field, were deduced. From I-V and $\mathrm{P}-\mathrm{V}$ characteristics of the solar cell, optimal photovoltage and optimal photocurrent obtained at the maximum power point corresponding to a given operating point which is correlated to an optimal junction recombination velocity, were determined according to the magnetic field. By means of the relation between the photocurrent density and the photoconductivity, the junction electric field has been determined at a given optimal junction recombination velocity.
\end{abstract}

\section{Keywords}

Bifacial Solar Cell, Photoconductivity, Junction Recombination Velocity, Magnetic Field, Electric Field

\section{Introduction}

The photoconductivity is due to the absorption of incident photons that create free charge carriers in the conduction band and/or in the valence band of a conducting material. The photoconductivity is one of the important quantities of 
solar cells or semiconductors [1] [2] [3]. It is a useful tool to study the properties of semiconductors like the recombination centers and the distribution of the deep levels states in the forbidden band. Many studies on the photoconductivity, have been utilized for determining a few intrinsic parameters of semiconductors in different regimes, as: in transient one [4] [5], where a relationship between the photoconductivity, the average lifetime and the generation rate of the photogenerated minority carriers has led to determine the density of states as function of the temperature; in steady-state [6]-[12], without an applied magnetic field, the effective lifetime of the minority carriers is determined and then the photoconductivity according to the temperature, the generation rate and the incident light intensity, what permits to determine the recombination density of states. While, with an applied magnetic field, the photoconductivity is, on one hand, as function of the magnetic field and the junction recombination velocity [13] and on the other hand, as function of the wavelength, the magnetic field and the incident power [14] [15]. By the use of the Fermi-Dirac distribution, it is demonstrated that the electrical conductivity of a silicon material decreased with the electric field [16]. Many researches have been carried out on the determination of the junction electric field by considering any more a magnetic field effect at a corresponding functioning point of a solar cell. That permits us to study, in this work, the photoconductivity of a bifacial silicon solar cell according to the magnetic field and the junction recombination velocity in order to determine the optimal operating point and the corresponding junction electric field.

\section{Theory}

\subsection{Mathematical Equations}

In Figure 1, an n+-p-p+ type of a bifacial silicon solar cell [17] [18], in 1D, is represented:

With $d$ and $H$ respectively the emitter thickness and the solar cell base thickness

We consider a constant magnetic field vector $\overrightarrow{\boldsymbol{B}}$ [19] [20] [21], that is applied following the $y$-axis perpendicularily to the solar cell plane.

So, the expression of the photoconductivity is given by [22]:

$$
\sigma_{p h}=\frac{q \mu_{o} \delta_{n}}{1+\mu_{o}^{2} B^{2}}
$$

where $q$ is the elementary charge of electron and $\mu_{o}$ the quasi-static mobility of the minority carriers without magnetic field.

One can see, in Equation (1) that the expression of the photoconductivity depends on the minority carriers' density $\delta_{n}$ and magnetic field. Thus, in steady-state, the minority carriers' density can be determined by resolving the following continuity equation:

$$
D_{n} \frac{\partial^{2} \delta_{n}}{\partial x^{2}}-\frac{\delta_{n}}{\tau}=-G
$$




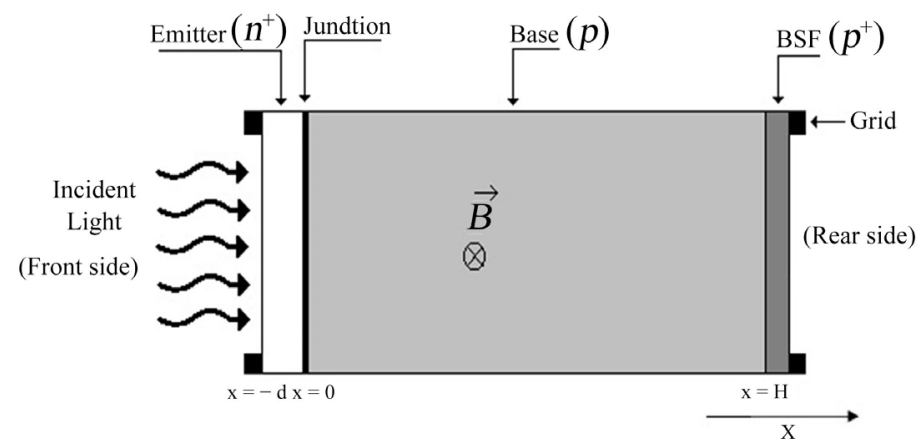

Figure 1. Schematic structure of a bifacial silicon solar cell under an applied magnetic field.

where $\tau$ is the average lifetime of the minority carriers in the base; $D_{n}$ is the minority carriers' diffusion coefficient which expression is given by:

$$
D_{n}=\frac{D_{o}}{1+\mu_{o}^{2} B^{2}}
$$

With $D_{o}$ being the minority carriers diffusion coefficient without applied magnetic field; $G$ the minority carriers generation rate [23] at the position $x$.

The expression of the minority carriers' generation rate, can be given as:

$$
G=\eta \sum_{i=1}^{3} a_{i} \mathrm{e}^{-b_{i} x}
$$

with $\eta$ being the solar number, $a_{i}$ and $b_{i}$ are coefficients deduced from modelling of the generation rate considered over all solar radiation spectrum under AM 1.5 .

The solution of Equation (2) can be written as:

$$
\delta_{n}=A \mathrm{e}^{-\frac{x}{L_{n}}}+C \mathrm{e}^{\frac{x}{L_{n}}}-\sum_{i=1}^{3} \frac{a_{i} L_{n}^{2} \mathrm{e}^{-b_{i} x}}{D_{n}\left(b_{i}^{2} L_{n}^{2}-1\right)}
$$

with:

$$
L_{n}=\sqrt{D_{n} \tau}
$$

and:

$$
\left(b_{i}^{2} L_{n}^{2}-1\right) \neq 0
$$

where $L_{n}$ is the minority carriers diffusion length in the base.

The coefficients $A$ and $C$ are determinated by the following boundary conditions [24] [25]:

- at the junction $(x=0)$

$$
\left.D_{n} \cdot \frac{\partial \delta_{n}}{\partial x}\right|_{x=0}=\left.S f \cdot \delta_{n}\right|_{x=0}
$$

- at the rear side $(x=H)$

$$
\left.D_{n} \cdot \frac{\partial \delta_{n}}{\partial x}\right|_{x=H}=-\left.S b \cdot \delta_{n}\right|_{x=H}
$$


where $S f$ and $S b$ are respectively the junction recombination velocity that defines the solar cell operating point and the back surface recombination velocity.

\subsection{The Photocurrent Density, Photovoltage and Solar Cell Output Power}

- The photocurrent density is related to the gradient of the photogenerated minority carriers at the junction and is given by:

$$
J_{p h}=\left.q D_{n} \frac{\partial \delta_{n}}{\partial x}\right|_{x=0}
$$

- The storage of the photogenerated minority carriers to the vicinity of the junction creates a photovoltage whose expression is given by the following Boltzmann equation:

$$
V_{p h}=V_{T} \ln \left(1+\frac{N_{B}}{n_{i}^{2}} \delta_{n}(0)\right)
$$

With:

$$
V_{T}=K T / q
$$

where $V_{T}$ is the thermal voltage at temperature $T=300 \mathrm{~K} ; N_{B}$ is the doping acceptor atoms in the base; $n_{i}$ is the intrinsic concentration of the minority carriers at temperature $T$ and $K$ is the Boltzmann constant.

- The solar cell output power is the energy supplied, at a time t, by the solar cell. Its expression is given by:

$$
P_{p h}=J_{p h} \cdot V_{p h}
$$

\section{Results and Discussions}

We present, in first, the photoconductivity profile, the $I-V$ and $P$ - $V$ characteristics and then in the second time, the determination method of the optimal operating point and junction electric field.

\subsection{Profile of the Photoconductivity According to the Junction Recombination Velocity}

In Figure 2, the photoconductivity versus the junction recombination velocity, is represented for different values of the magnetic field:

In Figure 2, we obtain the same behavior for the four curves of the photoconductivity versus junction recombination velocity. Considering curve 1 , without an applied magnetic field, in the interval of the junction recombination velocity $\left[0 \mathrm{~cm} \cdot \mathrm{s}^{-1} ; 2 \times 10^{2} \mathrm{~cm} \cdot \mathrm{s}^{-1}\right]$ where the solar cell operates in an open circuit situation, we note a high value of the photoconductivity that stays constant. This is related to a great photogeneration of the minority carriers around the junction where they cannot cross since they have not enough kinetic energy. However, in the interval $\left[2 \times 10^{2} \mathrm{~cm} \cdot \mathrm{s}^{-1} ; 5 \times 10^{5} \mathrm{~cm} \cdot \mathrm{s}^{-1}\right]$ that corresponds to a variable operating point of the solar cell, the photoconductivity decreases because the minor- 
ity carriers gradually gain a kinetic energy to across the junction. When the solar cell operates in a short-circuit situation, for a junction recombination velocity Sf $\geq 5 \times 10^{5} \mathrm{~cm} \cdot \mathrm{s}^{-1}$, the photoconductivity is lesser than of in open circuit one. In this situation, the minority carriers have enough kinetic energy to across the junction and there is no more new photogenerated carriers.

\subsection{I-V Characteristics}

The $I$ - $V$ characteristics, for different values of the magnetic field, are given in Figure 3:

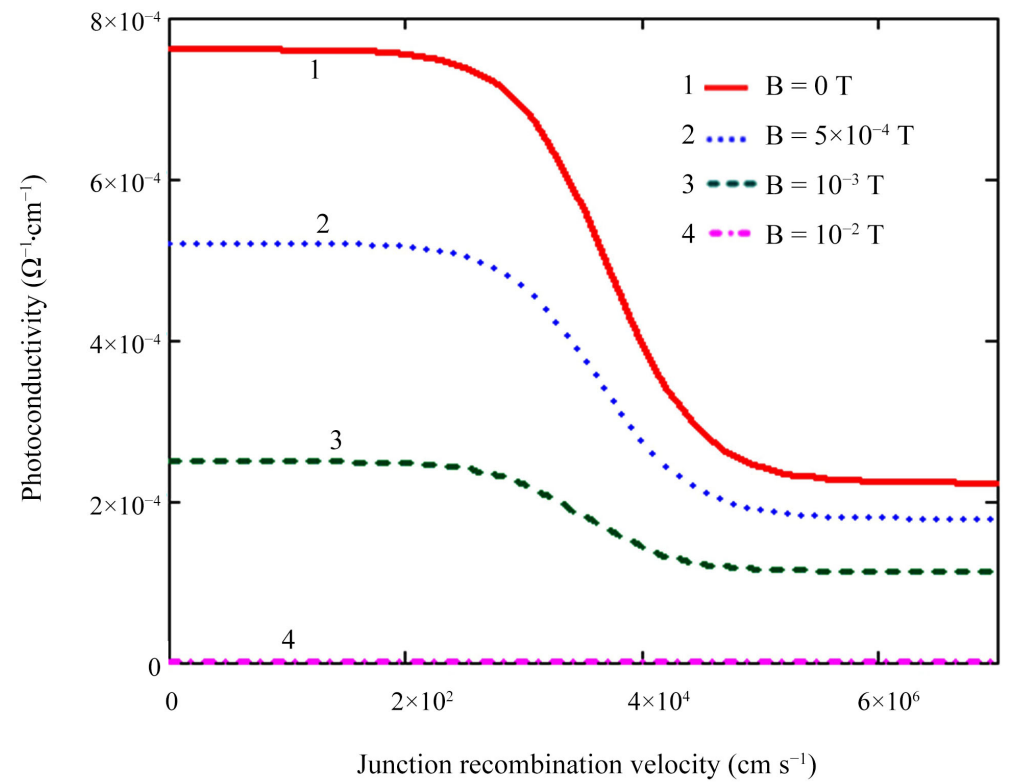

Figure 2. Photoconductivity versus junction recombination velocity for different magnetic field values.

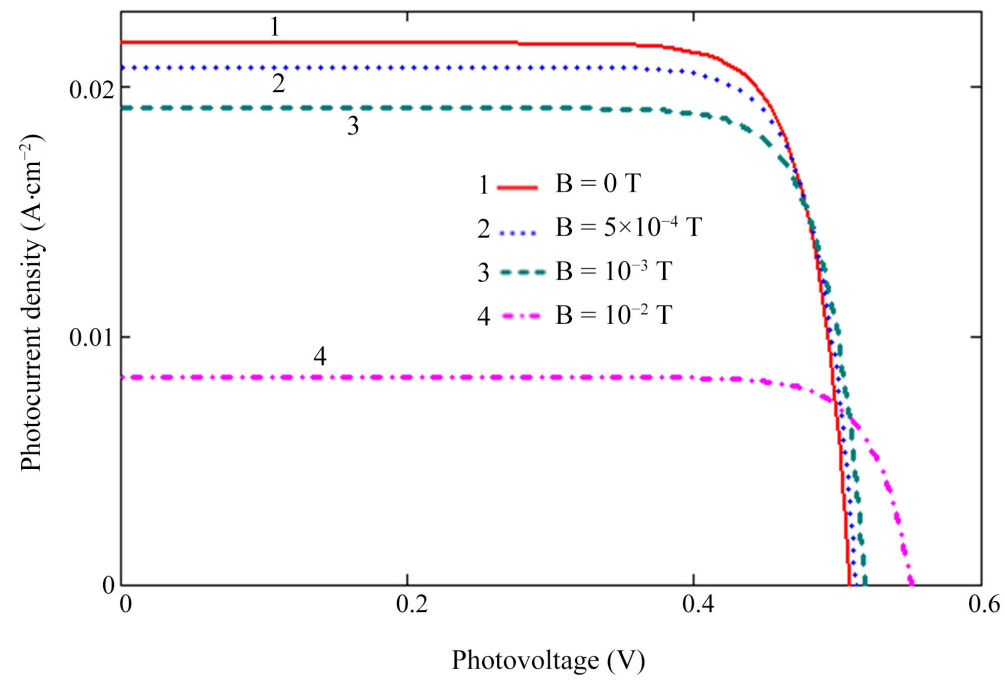

Figure 3. Photocurrent density versus photovoltage for different magnetic field values. 
The four curves in Figure 3, exhibit the same behaviour. For a low photovoltage, the photocurrent is maximum and corresponds to the short-circuit photocurrent: there is no significant storage of the photogenerated minority carriers in the vicinity of the junction since the majority of these carriers have acrossed the junction. This situation occurs when the junction recombination velocity is very high while the load is low. However, in the open circuit situation of the solar cell, there is a significant storage of the minority carriers in the vicinity of the junction; this implies a high photovoltage which corresponds to the open circuit photovoltage and where the photocurrent is closed to zero. With a magnetic field, the amplitude of the photocurrent decreases while the photovoltage increases. We note that the magnetic field slows down the photogenerated minority carriers in the vicinity of the junction or deviates them from their initial direction. We note that the operating point of the solar cell depends on the load and the magnetic field. That is why this operating point will impact on the output power delivered by the solar cell.

\subsection{P-V Characteristics}

In Figure 4, the output power versus the photovoltage, for different values of the magnetic field, is represented:

The curves of the $P$ - $V$ characteristics, in Figure 4, show similar profiles. For a given curve and its corresponding value of magnetic field, we note three zones:

1) a first zone which corresponds to the short-circuit situation of the solar cell and where the power increases linearly with the photovoltage. The fit of the curve give positive slope that corresponds to a short-circuit photocurrent;

2) a second zone corresponding to the open circuit situation of the solar cell where the power decreases with the photovoltage: it is like a solar cell that loses an energy and cannot work correctly since the load is high;

3) a third zone which is curvated and located between the two zones mentioned above, where the solar cell operates around the optimal power point.

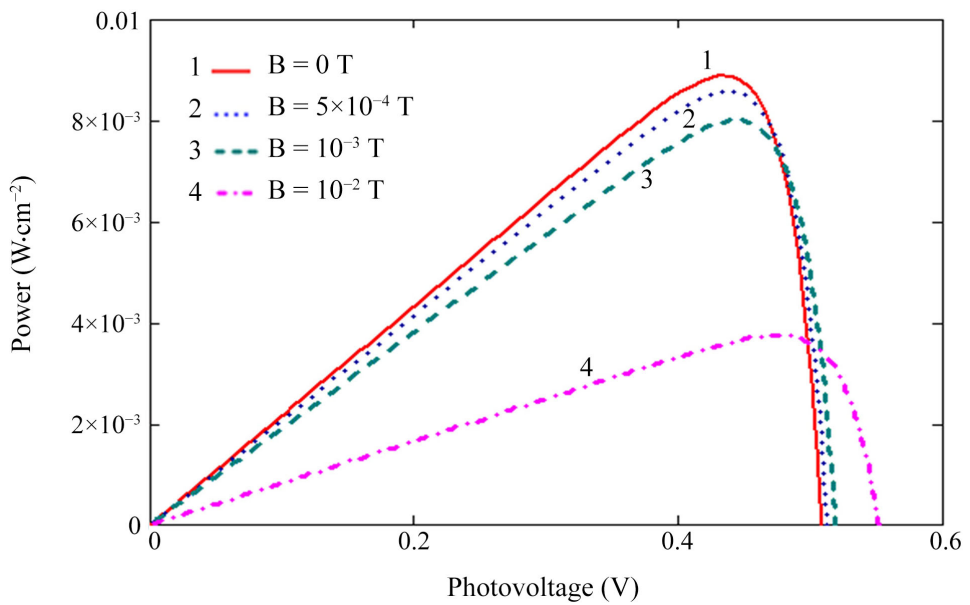

Figure 4. Power versus photovoltage for different magnetic field values. 
The increase of the magnetic field decreases the power delivered by the solar cell on the load.

From $I-V$ and $P-V$ characteristics, we propose a method for determining the optimal power operating point and the corresponding junction electric field.

\subsection{Determination Method of the Optimal Operating Point and Junction Electric Field}

We give, at first, a relationship between the photocurrent density and the electric field, as followed:

$$
J_{p h}=\sigma_{p h} \cdot E
$$

where $E$ is the electric field.

In Figure 5, a graphical determination method of the optimal operating point, is presented [26].

The $I-V$ and $P-V$ characteristics, in Figure 5, are given without an applied magnetic field and for an operating point that varies from the open circuit situation to the short-circuit one. The operating point is as function of the junction recombination velocity which is linked to the load. A high load corresponds to a low junction recombination velocity while a feeble load corresponds to a high junction recombination velocity. Therefore, there is a load that is linked to an operating point in which the solar cell delivers a maximum power $P_{\max }$. This operating point of the solar cell, which corresponds to the maximum power, is called the optimal operating point and is showed in Figure 5 by the point $P$. The $\mathrm{P}$ point projection on the photovoltage axis, gives us the optimal photovoltage $V_{\text {phop }}$. Its extension on the I-V characteristic, gives a point whose projection on the photocurrent axis allows us to obtain the optimal photocurrent $J_{\text {phop }}$. With the obtained values of $V_{\text {phop }}$ and $J_{\text {phop }}$, we deduce the optimal junction recombination velocity $S f_{o p}$ which corresponds to the optimal operating point of the solar cell.

In Figure 6, the optimal junction recombination velocity $S f_{o p}$ and the optimal photoconductivity $\sigma_{\text {phop }}$, are determined:

The photoconductivity and the photocurrent density are represented on both sides, in Figure 6, according to the junction recombination velocity which defines the operating point of the solar cell. From the curve of the photocurrent density versus the junction recombination velocity, the optimal photocurrent $J_{\text {phop }}$ gives a point whose projection on the junction recombination velocity axis, allows us to obtain the value of $S f_{o p}$. With the curve of the photoconductivity versus the junction recombination velocity, we determine the optimal photoconductivity $\sigma_{\text {phop }}$. From the values of $J_{\text {phop }}$ and $\sigma_{\text {phop }}$ that are obtained, we deduce the value of the junction electric field $E$ for a given optimal operating point of the solar cell.

These steps of determining all of the optimal values corresponding to an optimal operating point, are considered for different magnetic field values. What permits us to give Table 1 below: 


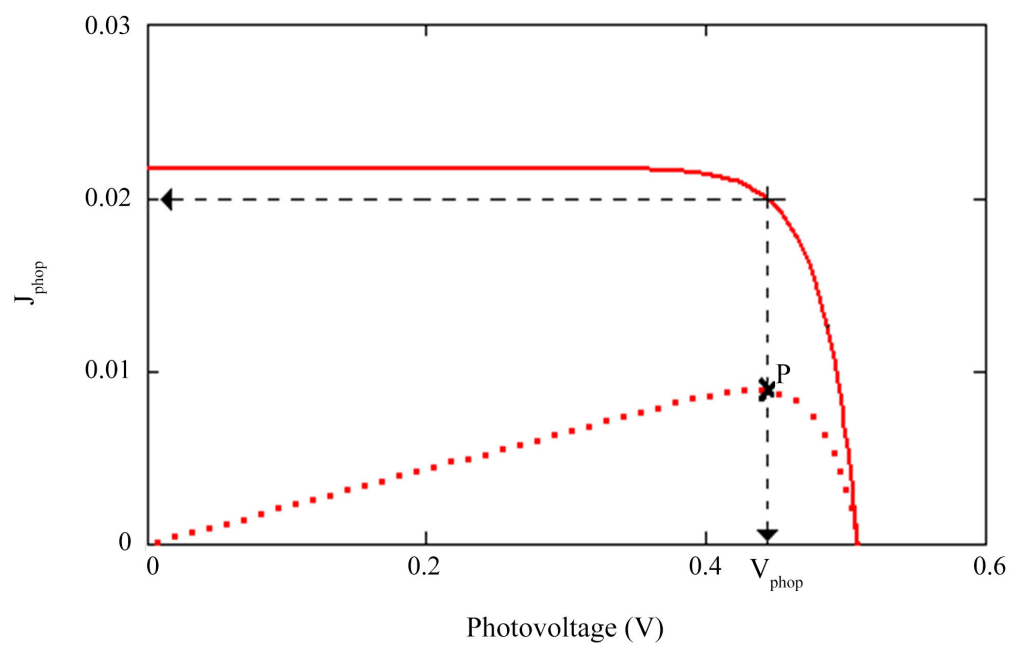

Figure 5. Determination of the maximum power and the optimal photocurrent for a given magnetic field $B=0 \mathrm{~T}$.

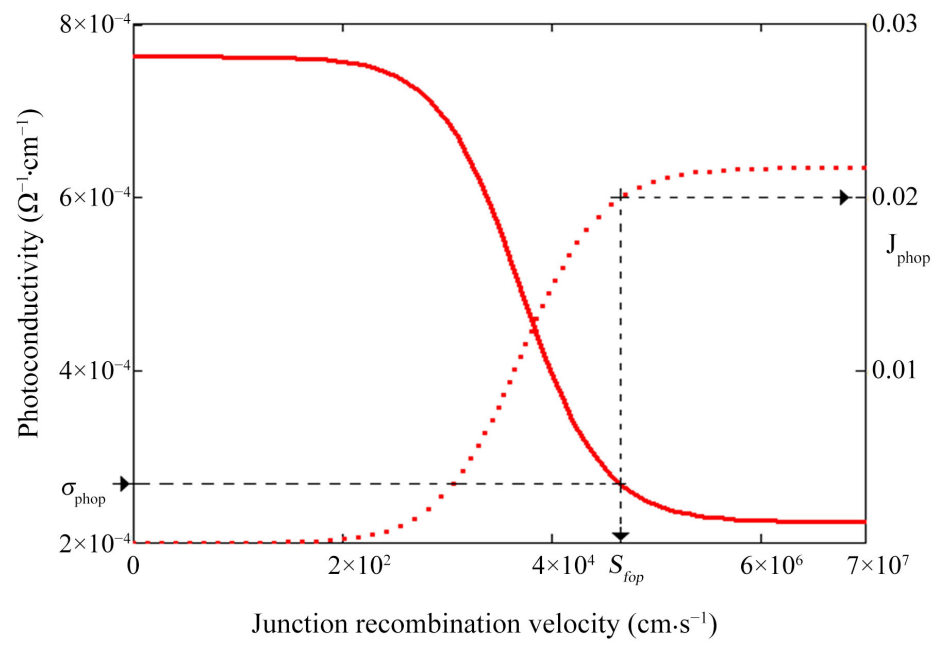

Figure 6. Determination of the optima of junction recombination velocity and photoconductivity for a given magnetic field $B=0 \mathrm{~T}$.

Table 1. A few values of $V_{\text {phop }}, J_{\text {phop }}, P_{\max }, S f_{\text {op }}, \sigma_{\text {phop }}$ and $E$.

\begin{tabular}{ccccc}
\hline$B(\mathrm{~T})$ & 0 & $5 \times 10^{-4}$ & $10^{-3}$ & $10^{-2}$ \\
\hline$V_{\text {phop }}(\mathrm{mV})$ & 438 & 442 & 445 & 480 \\
$J_{\text {phop }}\left(\mathrm{A} \cdot \mathrm{cm}^{-2}\right)$ & 0.02 & 0.019 & 0.018 & 0.0078 \\
$P_{\max }\left(\mathrm{mW} \cdot \mathrm{cm}^{-2}\right)$ & 8.76 & 8.39 & 8.01 & 3.74 \\
$S f_{\text {op }}\left(\mathrm{cm} \cdot \mathrm{s}^{-1}\right)$ & $3 \times 10^{5}$ & $2 \times 10^{5}$ & $1.8 \times 10^{5}$ & $2 \times 10^{4}$ \\
$\sigma_{\text {phop }}\left(\Omega^{-1} \cdot \mathrm{cm}^{-1}\right)$ & $2.59 \times 10^{-4}$ & $2.02 \times 10^{-4}$ & $1.22 \times 10^{-4}$ & $1.78 \times 10^{-6}$ \\
$E\left(\mathrm{~V} \cdot \mathrm{cm}^{-1}\right)$ & 77 & 94 & 147 & 4382 \\
\hline
\end{tabular}

Both the optimal photovoltage and the junction electric field increase with the magnetic field because the magnetic field slows down and stores the photocreated minority carriers in the vicinity of the junction. The optimal photocur- 
rent density, the maximum power, the optimal junction recombination velocity and the optimal photoconductivity decrease with the magnetic field. Therefore, we note that, a high photoconductivity corresponds to an ohmic solar cell in which the electric field is weak since the recombination of the minority carriers in the bulk of the solar cell becomes weak. However, a low photoconductivity corresponds to a nonohmic solar cell in which the electric field and the minority carriers' recombination in bulk are high. From these results we note a degradation of the intrinsic parameters of the solar cell when a magnetic field is applied.

\section{Conclusions}

A theoretical study has been done on a bifacial silicon solar cell. The photoconductivity, the photocurrent density and the photovoltage are determined according to the junction recombination velocity and the magnetic field. From the $\mathrm{I}-\mathrm{V}$ and $\mathrm{P}-\mathrm{V}$ characteristics, the maximum power operating point of the solar cell, the optimal photocurrent and the optimal photovoltage were determined for different magnetic field values. The optimal junction recombination velocity, the optimal photoconductivity and the electric field were deduced. The photovoltage and the electric field increase with the increase of magnetic field while the maximum power and the photoconductivity decrease. These obtained results were satisfactory and matched to other works in the research field. A simple method is utilized to determine the optimal operating point and it can be used for photovoltaic panels in order to optimize the load.

The flux of the incident photons and the temperature of the solar cell were assumed to be constant. The degenerated density of states and the change of the energy gap with the magnetic field were not taken into account in this work. In the future, we will consider these new elements in order to improve the analysis of our results. We intend to propose an algorithm to determine the maximum power and the corresponding electric field at the optimal operating point of the solar cell.

\section{Acknowledgements}

We acknowledge the Semiconductors and Solar Energy Laboratory and the Research team in renewable energies, materials and laser for supporting this work.

\section{Conflicts of Interest}

The authors declare no conflicts of interest regarding the publication of this paper.

\section{References}

[1] Bube, R.H. (1960) Photoconductivity of Solids. Wiley, New York.

[2] Joshi, N.V. (1990) Photoconductivity: Art, Science and Technology. Marcel Dekker, New York.

[3] Orton, J. (2004) The Story of Semiconductors. Oxford University Press, Oxford. 
[4] Adriaenssens, G.J., Baranovskii, S.D., Fuhs, W., Jansen, J. and Öktü, Ö. (1995) Physical Review B, 51, 9661-9667. https://doi.org/10.1103/PhysRevB.51.9661

[5] Belgacem, H. and Merazga, A. (2008) Solid-State Electronics, 52, 73-77. https://doi.org/10.1016/j.sse.2007.07.023

[6] Sinton, R.A. and Cuevas, A. (1996) Applied Physics Letters, 69, 2510-2512. https://doi.org/10.1063/1.117723

[7] Qiu, F., Xiang, J.Z., Kong, J.C., Yu, L.J., Kong, L.D., Wang, G.H., Li, X.J., Yang, L.L., Li, C. and Ji, R.B. (2011) Journal of Semiconductors, 32, 26-30. https://doi.org/10.1088/1674-4926/32/3/033004

[8] Qamhieh, N. and Adriaenssens, G.J. (2001) Journal of Non-Crystalline Solids, 292, 80-87. https://doi.org/10.1016/S0022-3093(01)00855-9

[9] Pal, R.K., Krishna, J., Agnihotri, A.K., Singh, C.P., Yadav, S. and Kumar, A. (2009) Chalcogenide Letters, 6, 29-34.

[10] Reis, F.T. and Chambouleyron, I. (2002) Journal of Non-Crystalline Solids, 299-303, 179-184. https://doi.org/10.1016/S0022-3093(01)01159-0

[11] Sharmin, M., Choudhury, S., Akhtar, N. and Begum, T. (2012) Journal of Bangladesh Academy of Sciences, 36, 97-107. https://doi.org/10.3329/jbas.v36i1.10926

[12] Schmidt, J.A., Longeaud, C. and Kleider, J.P. (2005) Thin Solid Films, 493, 319-324. https://doi.org/10.1016/j.tsf.2005.08.060

[13] Diao, A., Wade, M., Thiame, M. and Sissoko, G. (2017) Journal of Modern Physics, 8, 2200-2208. https://doi.org/10.4236/jmp.2017.814135

[14] Omar, M.S. and Abbas, T.A. (2010) Iranian Journal of Physics Research, 9, 99-102.

[15] Heisel, W., Bohm, W. and Prettl, W. (1981) International Journal of Infrared and Millimeter Waves, 2, 829-837. https://doi.org/10.1007/BF01007279

[16] Getinet, T. (2010) Indian Journal of Pure \& Applied Physics, 48, 192-195.

[17] Meier, D.L., Hwang, J.-M. and Campbell, R.B. (1998) IEEE Transactions on Electron Devices, ED-35, 70-78. https://doi.org/10.1109/16.2417

[18] Hüber, A., Aberle, A.G. and Hezel, R. (2001) 20\% Efficient Bifacial Silicon Solar Cells. 14th European Photovoltaic Solar Energy Conference, Munich, 22-26 October 2001, 1796-1798.

[19] Madougou, S., Made, F., Boukary, M.S. and Sissoko, G. (2007) Advanced Materials Research, 18-19, 303-312. https://doi.org/10.4028/www.scientific.net/AMR.18-19.303

[20] Thiam, N., Diao, A., Ndiaye, M., Dieng, A., Thiam, A., Sarr, M., Maiga, A.S. and Sissoko, G. (2012) Research Journal of Applied Sciences, Engineering and Technology, 4, 4646-4655.

[21] Madougou, S., Made, F., Boukary, M.S. and Sissoko, G. (2007) Advanced Materials Research, 18-19, 313-324.

https://doi.org/10.4028/www.scientific.net/AMR.18-19.313

[22] Kittel, C. (1972) Introduction à la Physique de l'étatsolide. Université, Dunod, 284-286.

[23] Mohammad, S.N. (1987) Journal of Applied Physics, 61, 767-772. https://doi.org/10.1063/1.338230

[24] Sissoko, G., Sivoththanam, S., Rodot, M. and Mialhe, P. (1992) Constant Illumination-Induced Open Circuit Voltage Decay (CIOCVD) Method, as Applied to High Efficiency Si Solar Cells for Bulk and Back Surface Characterization. 11 th European Photovoltaic Solar Energy Conference and Exhibition, Poster 1B, Montreux, 12-16 
October 1992, 352-354.

[25] Sissoko, G., Nanéma, E., Corréa, A., Biteye, P.M., Adj, M. and N’Diaye, A.L. (1998) Silicon Solar Cell Recombination Parameters Determination Using the Illuminated I-V Characteristic. World Renewable Energy Congress, Florence, 20-25 September 1998, 1847-1851.

[26] Lipinski, M. (1996) Opto-Electronics Review, 4, 129-133. 